\title{
Interdisciplinary Approaches and Strategies from Research Reproducibility 2020: Educating for Reproducibility
}

\author{
Melissa L. Rethlefsen ${ }^{1}$, Hannah F. Norton ${ }^{2}$, Sarah L. Meyer ${ }^{2}$, Katherine A. MacWilkinson ${ }^{3}$, \\ Plato L. Smith $\mathrm{II}^{4}$, Hao $\mathrm{Ye}^{2}$ \\ ${ }^{1}$ Health Sciences Library \& Informatics Center, University of New Mexico; ${ }^{2}$ Health Science Center Libraries, George A. Smathers \\ Libraries, University of Florida; ${ }^{3}$ Conference Department, University of Florida; ${ }^{4}$ George A. Smathers Libraries, \\ University of Florida
}

\begin{abstract}
Research Reproducibility: Educating for Reproducibility, Pathways to Research Integrity was an interdisciplinary, virtual conference hosted virtually by the University of Florida in December 2020. It brought together educators, researchers, students, policy makers, and industry representatives from across the globe to explore best practices, innovations, and new ideas for education around reproducibility and replicability. Emphasizing a broad view of rigor and reproducibility, the conference touched on many aspects of introducing learners to transparency, rigorous study design, data science, data management, replications, and more. Transdisciplinary themes emerged from the panels, keynote, and submitted papers and posters. The identified themes included lifelong learning, cultivating bottomup change, "sneaking in" learning, just-in-time learning, targeting learners by career stage, learning by doing, learning how to learn, establishing communities of practice, librarians as interdisciplinary leaders, teamwork skills, rewards and incentives, and implementing top-down change. For each of these themes, we share ideas, practices, and actions as discussed by the conference speakers and attendees.
\end{abstract}

Keywords: reproducibility, research integrity, interdisciplinary education

\section{INTRODUCTION}

Cultivating the next generation of researchers is a key component of fostering a culture of research integrity. Research integrity training primarily focuses on the responsible conduct of research (RCR), covering a wide swath of topics designed to impart unto trainees the principles of research rigor, ethical frameworks for evaluating different options, and practices that uphold community standards (1). Using these values and ethical frameworks, trainees can be expected to make better choices when confronted with difficult choices (2). However, one area that RCR training has covered less, if at all, is reproducibility.

Reproducibility and replicability are cornerstones of research integrity and science, ensuring that experimental procedures can be performed by others, and that the steps and decisions involved in published results are presented openly and honestly. Recent concerns about the reproducibility "crisis" have led to increased public, scientific, and governmental scrutiny on this topic (3-6). To date, concerns about research reproducibility have surfaced in dozens of disciplines, from economics to neuroscience $(7,8)$. Individual researchers and research results are often publicly questioned about reproducibility 
concerns, shaping widespread, public conversations about the integrity and even validity of the scientific process (9-13). Though outright fraud and serious misconduct remain rare, large-scale replication projects, surveys, and statistical analyses of published research confirm that a much larger proportion of scientists engage in "questionable research practices" (14), such as data dredging (p-hacking) or HARKing (hypothesizing after results are known) $(15,16)$.

Simple solutions for achieving reproducible and replicable science are inherently impossible because of the sheer complexity of science. Indeed, culture change can include many different actions, from multiple stakeholders, who each have different priorities (17). Without a broad consideration of the interrelated components of the research enterprise, initiatives may "fail because they are too focused on one aspect of the system, commonly technology, and fail to analyse and understand the complex interdependencies that exist" (18). Even for a specific problem, such as making research data findable, accessible, interoperable, and reusable/reproducible (FAIR), a multitude of stakeholders may be involved, including the University, Office of Research, Research Compliance Office, Information Technology (IT) Department, Researchers, Academic Units, and Library (19). Bringing together the perspectives from all of these stakeholders is essential to ensure successful culture change.

Similarly, although reproducibility cuts across all research disciplines, the issues and ideal solutions may be discipline-agnostic, just as in other facets of RCR education. For that reason, coding and data sciencefocused courses have emerged in this space; they are helpful to nearly all researchers (20-22). Though these courses can provide some of the essential tools and skills, they do not answer the larger questions about how institutions can and should support students navigating situations involving questionable research practices or other ethical and policy questions. How to best train researchers in this area is still unknown. Whereas students, trainees, and other early career researchers look to established mentors for guidance in identifying and tackling research projects, these experts may be less familiar about navigating reproducibility and RCR-researchers at all stages can benefit from education and skill building.

For these reasons, the University of Florida Health Science Center Libraries, in partnership with the George A. Smathers Libraries, hosted a 2-day virtual conference on December 2-3, 2020 to focus on the problem of reproducibility education. We brought together representatives from multiple stakeholder groups and spanning disciplines to discuss challenges, opportunities, and existing programmatic examples around reproducibility education. The conference, Research Reproducibility 2020: Educating for Reproducibility (23), was funded by the U.S. Department of Health \& Human Services' Office of Research Integrity. In this paper, as in the conference, we purposefully use a very broad definition of reproducibility that encompasses computational reproducibility, inferential reproducibility, methods reproducibility, replicability, rigor, and transparency.

\section{METHODS}

The meeting was designed to meet three main objectives: 
1. Increase knowledge of educational approaches to foster research integrity through reproducibility

2. Provide attendees with networking opportunities for brainstorming and collaboration and provide a forum for discussion

3. Build awareness of reproducibility as a component of research integrity and responsible conduct of research education

\subsection{Participants}

The conference was designed to bring together educators, administrators, students, and researchers from a wide range of disciplines and institutions. Since the conference was initially planned as an inperson event, there was also a local and regional focus in bringing together participants and speakers from across the University of Florida (UF), the State of Florida, and nationally. With the change to a 2day virtual conference, due to COVID-19 restrictions, we were able to dramatically increase the number of participants from outside of Florida.

429 attendees registered for the virtual conference, including participants from 21 countries and 40 U.S. states, plus Washington, DC. In addition, registrants represented 182 unique organizations and institutions, including academic institutions, non-profit organizations, and for-profit companies. Conference speakers and presenters came from the United States, Taiwan, England, Germany, and Spain and represented a myriad of disciplines, including behavioral science, bioethics, biology, biostatistics, biomedical informatics, clinical psychology, data science, economics, geographical data science, human and molecular genetics, information science, linguistics, medicine, metabolomics, neuroscience, open science, psychology, public health, and social work. Presenters ranged in career stage from undergraduate students to senior faculty, as well as staff from nonprofits and for-profit companies.

\subsection{Conference Design}

The conference schedule encompassed a variety of modalities and types of content, including a keynote, three moderated panels, submitted oral paper presentations and lightning talks, an open poster session, an unconference/breakout discussion session, and scheduled times for casual conversations and discussions. We used the online conference platform, Remo, which enabled attendees to "sit" at virtual tables with up to six people each, to mimic small gatherings at an in-person conference. All attendees at a table could converse with each other via video, audio, and group chat, in addition to sharing content, such as posters during the poster session. The platform also enabled content to be broadcast to all attendees, such as the keynote, panels, and oral presentations, as well as a general chat that attendees could use to ask for technical help or find colleagues.

Day One: After brief opening remarks by the conference host, Brian Nosek presented the opening keynote. His talk centered around the current culture in research and how it might be shifted. After the keynote were two concurrent paper sessions with six total submitted talks. After a brief break, the conference resumed with a moderated panel session focusing on undergraduate and graduate student education in reproducibility with speakers from 
neuroscience, linguistics, and biostatistics. A second set of concurrent paper sessions followed, with six additional talks. Directly following the papers were two concurrent lightning talk sessions, designed for poster presenters to highlight their posters before the poster session. The day concluded with the poster session.

Day Two: After introductory remarks by the conference host and sponsor, there was a second moderated panel discussion that focused on student experience and support for reproducibility. This wide-ranging panel, consisted of two students and two research integrity officers and covered topics including dealing with principal investigators who violate research ethics, places where students can go for help, and how students could be better prepared to conduct reproducible research. The last moderated panel, with speakers from economics, academic libraries, and data science/management organizations, focused on approaches to develop different types of reproducibility education for post-graduate and professional education. Throughout the conference, attendees were encouraged to submit their discussion topics for the unconference-style breakout discussions after the two panels. The day concluded with a virtual mixer to help continue discussions and conversations.

Moving to an online format after planning for an in-person conference required significant reassessment and assistance from our UF Conference Department event manager. It necessitated analysis of different online platforms to determine what met our conference goals, and required setting up a more robust conference website with speaker profiles, an abstract submission portal and collection site, and a more detailed conference agenda. As noted above, we used the Remo virtual platform, as it provided a similar interactive and engaging experience for conference attendees and speakers, especially fun and personalized networking during breaks and unconference discussions. It did require training, however; each presenter and session moderator was provided one-on-one training to ensure that the content delivery was translated well over a virtual learning environment. Session moderators helped to introduce each presenter, facilitate questions during the presentations, monitor time, and engage with the attendees, with all logistics supported by UF Conference Department staff and Remo technical support.

In addition to having the live panel and paper presentations, the virtual platform integrated with the UF Health Science Center Libraries YouTube Channel to livestream presentations both days of the conference. We also recorded each session and edited and uploaded session videos to the YouTube Channel and linked them on the conference website for post-event viewer access. Post-event data from the conference platform included all submitted attendee questions from keynote, panel, and paper presentations; the general event chat; attendee and speaker log-in dates; and total time spent in the platform, plus session video recordings.

A complete agenda, invited speaker biographies, links to conference videos, and all abstracts for submitted content are available on the conference web site (23).

Below, we reflect on themes and best practices revealed through the speaker sessions, academic posters, and discussions that took place throughout the two days of the conference. The themes are 
organized using the Center for Open Science's "Strategies for Culture Change," introduced to the attendees by the conference's keynote speaker, Brian Nosek, Co-Founder \& Executive Director, Center for Open Science $(17,24)$.

\subsection{Analysis of Themes}

To identify themes, planning team members reviewed all of the videos and content produced from the conference, individually for submitted content and in pairs for the keynote and panels. Each team member identified themes and topic areas that they noticed individually. The group came together for multiple discussions to identify and refine common themes. The themes below do not encompass every component of the conference, but rather strive to highlight interdisciplinary approaches and topics discussed by multiple presenters.

\section{RESULTS}

\subsection{Make It Possible}

\subsection{Practice Lifelong Learning.}

Across panels educators described motivators to embrace, embed, and promote open science practices to "make research credible" $(25,26)$. Approaches such as, embracing lifelong learning, embedding into educational content and promoting reproducible practices in writing and authorship are driven by an ability to foresee what is possible and act to make it possible. Panelists described leading by example to exemplify and foster an expectation for reproducible research practices. One speaker, Rachel HayesHarb, summed these up as "intentions to make our own research more reproducible" (26).

\subsection{Cultivate Bottom-up Change.}

Panelists described cultivating bottom-up support for reproducible education with localized approaches to embed and promote. For instance, as learners explore new knowledge, speakers described efforts to embed an infrastructure that promotes and instills open science research practices. An approach described by one panelist, Brian Avery (26), is the value of identifying colleagues to partner with on the development of reproducible education to "bring it out of isolation." Hayes-Harb explained students are the cornerstone of educational institutions and their expectations for social justice, transparency, and to make science trustworthy should be decision drivers (26). For instance, leading by example, mentoring, embracing learners' values and promoting them as change agents to expect and enable reproducible research cultures initiates bottom-up change $(25,26)$.

\subsection{Sneak in Learning.}

An emerging discussion in both educator panels was intentional integration of reproducible training into existing education. Two speakers, for instance, working with undergraduate students described integrating replication studies into existing curriculum $(25,26)$. Educators explained they do not seek 
permission or support to restructure traditional methods, instead they restructure, and "sneak" reproducible practices in and justify positive outcomes afterward (26). These "sneak-in" approaches offer opportunities to embed, tailor and align with discipline-specific research norms and teach applicable skills and tools. Student speakers expressed a preference for an embedded research reproducibility infrastructure (27).

Speakers working with undergraduate and graduate students were asked to discuss managing challenges with introducing and garnering support for new content. Educators across disciplines favored an approach that restructures traditional methods, rather than adding or removing content (26). For example, this could include using automated tools to calculate a t-test result, rather than traditional, by hand methods (26). Student panelists also expressed a preference for restructuring and perceived addon approaches diminish the value of reproducible research practices (27).

\subsection{Just-in-Time Learning.}

One speaker, Tracy Teal, explained that many open science practices focus on computational tools and users' past disempowered experiences with technology can negatively bias their perceptions (25). To address this challenge, educators teaching across career stages described creating positive open science learning environments and just-in-time approaches to training are important (28). Positive learning environments make it possible to build confidence, improve self-awareness, and enhance quality of life (e.g., reduce stress and increase efficiency). Just-in-time approaches such as those used in the Data Carpentries workshops ensure training content is accessible, approachable, aligned, and applicable; this promotes learners' confidence and satisfaction (25).

\subsection{Make It Easy}

\subsection{Target Learning by Career Stage and Where Learners Are.}

Speakers and presenters emphasized that general concepts around reproducibility are interdisciplinary, that a myriad of skills are required to make research reproducible, and that more detailed, disciplinespecific knowledge is required as students and researchers embark on their career paths. One of the lightning talk/poster presenters, Elizabeth Wickes (28), summed up the dilemma, saying that people at all levels of their careers need to have "on-ramps to learning." She noted that while introducing skills and concepts to people early may be ideal, middle and senior career individuals want to learn, but educators need to approach them with a growth mindset. Overall, a clear theme emerged that tailoring educational efforts to different career stages and specific audiences was key to engaging learners where they are.

Several presenters worked directly with undergraduate students, including working with them to conduct replication studies as part of coursework and in the lab. Central to the idea of educating undergraduates was that these students were at a point of undiminished idealism and could not only learn skills quickly, but if taught concepts around reproducibility, would carry them as part of how they always think about research in the future. Reaching undergraduates can help address research culture 
problems by "embedding research skills and sensibilities development within the responsible conduct of research and open science values and practices" from the beginning, enabling a bottom-up approach to shifting culture (26).

Two speakers specifically addressed using and conducting replication studies as a way to engage undergraduate researchers $(26,29)$, and one trio of undergraduate researchers who conducted a replication study as part of one of these courses presented about their research (30). Undergraduates are a particularly good group to target for these opportunities, because they can simultaneously build their experimental knowledge and scientific reasoning while also learning some of the theory and background behind the experiment.

Many more presenters addressed educating graduate students about reproducibility, both within master's degree and doctoral degree programs, generally in interdisciplinary settings. This included discussion about credit courses $(25,26,31,32)$, theses (33), and workshops (34). Education focused on graduate students was more frequently designed to build specific skill sets, including version control, programming, and data cleaning. Others required their graduate students to produce computationally reproducible research as part of their course-based projects or theses. One presenter also spoke to alternate means of introducing reproducibility into a graduate thesis process, by including online training and self-assessments of their theses' reproducibility as requirements (33). Several speakers noted graduate students were at a key stage of needing to learn how to learn, so that teaching them how to access and use resources to teach themselves was critical and well-received by students.

Targeting graduate students can help to spread knowledge, skills, and culture. One set of presenters specifically noted that training graduate students had an additional outcome; the students' learning influenced their faculty advisors' interest in learning about reproducibility concepts and skills (31). Richard Ball noted that Project TIER's new pilot project was to work with graduate students who were teaching assistants and would work directly with undergraduates. Not only would that training benefit the undergraduates, but could also influence how the graduate students being trained conduct their own research (25).

Another commonly targeted demographic was early career researchers, who need and want education, but who have greater limitations on their ability to participate in longitudinal or time-intensive training than undergraduate and graduate students. Multiple ways of engaging early career researchers were discussed, including ReproducibiliTea-style journal clubs that require limited time commitments $(25,31$, $35)$, multi-hour to multi-day workshops $(25,36)$, credit courses $(26)$, and fellowship-style programs that require more time and effort, or may require the faculty member to obtain release time $(25,37)$.

There are benefits to targeting early career researchers. Fellows programs that recruit early career researchers may grow researchers who become open science and reproducibility advocates at their local institutions, for instance. Another speaker, Matthew Gurka, who taught a six-week course focused on reproducibility to a range of learners, including early career clinical researchers, noted that the course was an opportunity to give future National Institutes of Health principal investigators an 
appreciation of building research teams with biostatisticians and data scientists and ensuring their research projects have resources and infrastructure to support reproducible research (26).

Targeting by career stage is not the only way to focus educational efforts. Another strategy identified by presenters was to build skills within a specific lab. For instance, a lab can establish good lab practices by creating detailed training protocols, lab manuals, predefined project templates and analysis plans, standard data management protocols, and other processes to ensure transparent reporting, data sharing, code sharing, and more. Two speakers shared their labs' processes to engage all members of the lab, including students who may not have been exposed to reproducibility principles elsewhere (38, 39). A lab setting can also be used to discuss ethical issues or help lab members learn new reproducibility skills (24).

\subsection{Balance Practical Skills Development with Conceptual Learning.}

While the instructional methods described throughout the conference were largely hands-on and application-based, speakers highlighted the importance of tying this practical learning to conceptual learning (26). One team of speakers added skills on writing transparently and without spin as well as creating transparent, reproducible code to an existing responsible conduct of research course covering the more theoretical elements of research integrity required by the National Institutes of Health (36). Additionally, several speakers grounded their own teaching and evaluation practices using theoretical frameworks of behavior change $(28,40)$.

\subsection{Learn by Doing.}

As discussed above, speakers from across various settings and disciplines found it effective and rewarding to teach learners the concepts of reproducible research by having them replicate studies themselves. This hands-on experience builds learners' experimental knowledge and refines their scientific reasoning skills. In the case of a psychology course, for example, experimental replication can help students produce new knowledge by building a bridge between the theory they are learning and the phenomenon they are attempting to describe (29). In attempting to reproduce studies, learners see first-hand what some of the barriers to reproducibility are: missing underlying data, inadequate description of workflows, and lack of access to specific software (41). Some of these and other data management issues may not be apparent at the small scale that early researchers are familiar with (e.g. working with single spreadsheets, small datasets); learning by doing illuminates these bigger issues (26). Performing replications led learners to reflect not only on the broader reproducibility issues facing research, but on their own practices. In their workshop for NIH researchers, for instance, Zaringhalam and Federer (41) noted that participants saw that their own papers are not reproducible and noted in evaluations that this will affect how they approach their research in the future.

\subsection{Learn How to Learn.}

Several speakers identified metacognition or learning to learn as another key to learners moving forward as critical thinkers and thoughtful researchers. In a data science course for biomedical students, 
for example, Lapato and Exner (31) added a session on metacognition, spending the time to teach learners how to teach themselves and think about how they would approach self-guided learning in the future. Learning to learn was mentioned in the undergraduate/graduate education panel as a step in helping students become independent critical thinkers (26). Likewise, at the student experience panel, speakers discussed the benefits to students teaching others about reproducible research concepts even as they are still learning themselves (27).

\subsection{Make It Normative}

\subsection{Establish Communities of Practice.}

Several speakers discussed the importance of communities of practice, or groups of people who meet regularly to practice previously learned skills, acquire new knowledge through peer-teaching, and socialize with colleagues $(25-27,35)$. Communities can operate in different modalities, including inperson and virtual. As noted by the speakers, these communities make regular practice and growth into a social experience and can help to maintain and expand participant capabilities. They can also accelerate the adoption of new approaches, by normalizing ways of thinking and doing within a group of like-minded peers.

Across the panels (25-27), multiple benefits to communities of practice were mentioned:

- Communities of practice can reinforce training that may have been initially acquired in more formal settings, as well as fill in learning gaps that can result from short-form training activities, such as workshops or boot camps.

- With rapid development of new tools and platforms for reproducibility and open science, communities of practice provide a mechanism for sharing information rapidly, e.g. code review for computational research (26).

- As a social group of peers, communities of practice enable individuals to discuss lab culture and ethical issues in a low-intensity setting, without the fear of retaliation or conflict that may occur if issues are brought up directly as a confrontation or reported to, e.g., ombudspeople or university research integrity offices.

Even with these benefits and interest, recruiting interest in communities of practice and sustaining their activities can be challenging. As might be expected, ReproducibiliTea journal clubs, discussed by Lapato and Exner (31), Chen et al (35), and Riegelman (25), have a reusable set of materials for jumpstarting local chapters. Although some decrease in attendance over a semester is natural, most attendees are highly motivated and small incentives such as providing food and tea can be helpful. In addition, framing activities as practices that make research easier and more effective can be more attractive to audiences than focusing on reproducibility explicitly (25). 


\subsection{Librarians as Interdisciplinary Leaders.}

Librarians in academic libraries and health sciences libraries are creating effective, interdisciplinary opportunities for educating their campuses and beyond about reproducibility and open science concepts and skills. Multiple speakers throughout the conference including librarians, researchers, educators, and students mentioned the role of librarians as early leaders and collaborators in their institutions. Several speakers mentioned libraries' role as an educational hub on campuses, serving as touchpoints for interdisciplinary skills and researchers. It was also emphasized that librarians' established partnerships and connections that enabled their educational programs to reach a broader audience $(25,31,34)$. This might be through individual consultations, library workshops, guest lectures or workshops for a specific class or cohort, hosting reproducibility-focused journal clubs, teaching credit courses, organizing code-a-thons aiming at reproducing published studies, or hosting other events (25, $28,31,34,41)$.

Librarians bring skills to the reproducibility space that others may not. Benefits and special knowledge and skills librarians concern organization, description (metadata), interoperability, taxonomies and ontologies, literature searching, systematic reviews, scholarly communication, and publishing/dissemination of research $(25,31)$. In addition, librarians have the ability to translate and explain complex reproducibility concepts in basic terms, due to their experience teaching interdisciplinary learners. For one presenter team, their work transitioning a general research skills credit course into one focused on reproducibility enabled them to create additional workshops designed to reach multiple audiences. Another presenter team noted that the partnerships they created enabled a suite of different educational offerings, from degree programs to a ReproducibiliTea-style journal club (31). Many of the librarians who presented or were discussed have been working in this space for many years, from co-leading Project TIER for twelve years to developing classes to support new requirements for rigor and reproducibility education for NIH training grants in 2016.

\subsection{Effective Teamwork Skills.}

Although reproducible research is often framed as an external benefit (i.e. the research is more reliable and useful to others), the practices that promote research reproducibility can also facilitate working on a research project alone or in collaboration (25). For example, documenting the biological materials using Research Resource Identifiers and tools like Addgene can make it easier to write up the methods section when publishing, and also helps ensure that data can be aggregated or compared across experiments that are done in different locations (e.g. a collaborator's lab) or at different times (e.g. a follow-up study exploring related questions) (42). Indeed Taylor and Marsiske (38) describe how principles in clinical trials can be implemented in single laboratory studies to provide a foundation for rigor, reproducibility, and transparency.

Establishing collective norms for research have impacts beyond just reproducibility, but also extend to better pathways for resolving conflict and other issues. As discussed in the student experience panel, there are differentiating ideas about which practices are acceptable, which are considered questionable 
research practices, and which may rise to the level of scientific misconduct (27). Creating and communicating shared norms in a collaboration or lab setting can head off these issues, and also enable more effective teamwork by clearly laying out responsibilities and rewards (including authorship order). In addition, these policies can also provide information on research processes, such as working with grants offices, IRB panels, systematic review services, and more. For trainees, this can be invaluable, as many do not start out being familiar with the intricacies of research (27).

Conference participants had a variety of suggestions on how best to implement teamwork policies. Wickes (28) recommends that practices be both specific and attainable, lest adoption of reproducibility be constrained by the lack of time and energy to engage with "best" practices. In the post-graduate education panel, Ball describes how concrete guidelines for data sharing from the American Economic Association have helped normalize reproducibility and motivate adoption by departments and lab groups (25). The role of faculty and PIs in setting and implementing these practices and policies is an important target for change.

\subsection{Make It Rewarding}

\subsection{Incentives and Rewards.}

In his keynote presentation, Nosek (24) identified rewards and incentives as another necessary pillar in creating broad cultural change and widespread adoption of reproducible research practices. One challenge here is that the commonly held values of transparency and research quality do not match the current rewards. Researchers are rewarded for high rates of publication, with positive results more likely to get published. Together, these rewards incentivize selective reporting and quantity of research output over quality, making questionable research practices more likely. Nosek (24) discussed two publisher and funder models designed to shift the incentives: registered reports and registered proposals. Registered reports shift peer review to occur after study design and before data collection, which incentivizes asking important research questions and developing good study design, rather than producing positive results or focusing purely on innovation. Registered proposals are reviewed by journal publishers and funders together and, when accepted, guaranteed both funding and publication, again incentivizing good study design and reproducible practices rather than positive results alone. Ideally, incentives should be offered at all levels so that institutional, publisher, funder, and professional society are able to effect meaningful change.

Several presenters in other parts of the conference made mention of the relevance of considering rewards and incentives specifically in education and training for reproducibility. For instance, Tracy Teal referred to a study where researchers reported most wanting training from that organization, even more than funding; this suggests that in some cases individuals may see intrinsic rewards in education around research practices (25). In a more specific example, another presenter described an online course on research transparency and reproducibility being coupled with a research transparency badge for those completing the course, which they could apply to their professional profile and/or email 
signature (40). Another set of presenters recruited participants to their various training offerings by emphasizing the benefits to the job market in having reproducible research skills (31).

\subsection{Make It Required}

\subsection{Implementing Top-Down Change.}

Though conference participants emphasized the important of bottom-up, grassroots efforts to spread change and influence communities, many speakers also noted the importance of top-down efforts by institutions, funding agencies, and journal publishers. For instance, Johnson and Goodman (36) noted that their workshops were designed to meet and exceed funder mandates for both responsible conduct of research and rigor and reproducibility education. They used the funder mandated education as a pathway to introduce better scientific writing practices and transparent coding skills. Multiple speakers commented on top-down federal mandates as a starting place for their work, though also noted that when educational efforts are construed as compliance or administrative issues, they are less valuable to learners, as they are often too abstract to translate to everyday efforts $(24,36)$. Lab-based discussion and reasoning about ethical practice involving authorship and data management policies and training, for instance, are lessons in research ethics closer to everyday concerns (24).

Improving transparency of research reporting through institutional and journal publisher policies was frequently discussed by speakers. Both reporting guidelines (i.e., CONSORT, ARRIVE 2.0, etc) and NIH rigor and reproducibility principles were lauded $(43,44)$, but speakers made it clear these are not as useful unless enforced by journal editors (45). Several presenters examined the transparency of published studies and preprints, which are still often poorly reported or of poor quality $(45,46)$. Ideas to implement templates for registered reports and interventions to improve reporting quality using automated messages to authors on how to improve their preprints were proposed $(47,48)$. Finally, speakers also suggested that departments and institutions should develop policies, including tenure and promotion policies, that require transparency and rigor (25).

\section{DISCUSSION}

To assess our intended outcomes, increased knowledge of educational approaches to foster research integrity through reproducibility, providing networking opportunities and a discussion forum, and building awareness of reproducibility as a component of research integrity and responsible conduct of research education, we conducted a brief evaluation. The response rate was poor (23/429 or 5\%). Thus, findings from the evaluation are not necessarily representative. Overall, $84 \%(n=19)$ of respondents said that the conference met all or most of their expectations and all but one respondent said they would attend the conference again $(95 \%, n=21)$. We asked the respondents to rate the keynote session, panels, and paper presentation sessions. All sessions were rated highly; the mean rating on a 5-point Likert scale ranged from 4.2 to 4.67 . $95 \%$ of respondents $(n=21)$ agreed that the conference was either extremely well-organized and planned or well-organized and planned. 
More informatively, we offered the opportunity for respondents to give us qualitative feedback about their learning and takeaways. Attendees appreciated the variety and quality of speakers, the diversity of the speakers' roles and disciplines, networking opportunities, and discussion components of the conference. One attendee said, "Hearing directly from faculty, staff, and students on these topics was wonderful." More importantly, multiple attendees noted that they took away ideas for reproducibility education that they would incorporate into their own teaching. For example, one attendee was inspired with new "ideas for training and workshops for grad students" and another was motivated to reach "out to audiences that [they] may not have thought about before to encourage reproducibility, starting as young as possible." Another noted that they "appreciated the information about culture change and will work to implement some of the ideas presented."

Hosting an interdisciplinary education conference enabled a wide range of participants that might not otherwise have met. We feel that we succeeded to fulfill our anticipated outcomes, and we look forward to our attendees' future engagement on this topic. Resources from the conference remain freely available and open for use, including videos of most presentations (49), posters and presentations (50), abstracts (51), and the collaborative notes from the sessions (52). Table 1 includes additional resources suggested by conference speakers. We hope that themes we have identified in this article and these products from the conference will inspire more educators to collaborate and come together to explore effective and innovative methods of reproducibility education.

Table 1. Select resources shared by speakers and attendees

\begin{tabular}{|l|l|l|}
\hline Name of Resource & Web Address & Audience/Purpose \\
\hline ReproducibiliTea Journal Clubs & $\begin{array}{l}\text { https://reproducibilitea.org/jour } \\
\text { nal-clubs/ }\end{array}$ & $\begin{array}{l}\text { Journal club leaders; provides } \\
\text { materials for reuse and ideas for } \\
\text { content }\end{array}$ \\
\hline $\begin{array}{l}\text { Berkeley Initiative for } \\
\text { Sransparency in the Social }\end{array}$ & https://www.bitss.org/ & $\begin{array}{l}\text { Learners, instructors; exercises, } \\
\text { workshops and programs, and } \\
\text { templates }\end{array}$ \\
\hline The Carpentries Curricula & $\begin{array}{l}\text { https://carpentries.org/worksho } \\
\text { ps-curricula/ }\end{array}$ & $\begin{array}{l}\text { Learners, instructors; provides } \\
\text { modular, step by step lessons } \\
\text { on data and code-related topics }\end{array}$ \\
\hline Center for Open Science & https://www.cos.io & $\begin{array}{l}\text { All; platform of open science } \\
\text { tools and initiatives, including } \\
\text { registered reports }\end{array}$ \\
\hline Reproducibility for Everyone & www.repro4everyone.org & $\begin{array}{l}\text { Learners, instructors; workshop } \\
\text { lessons that can be used and } \\
\text { adapted }\end{array}$ \\
\hline GitHub Learning Lab & https://lab.github.com/ & Learners; tutorials on using \\
\hline
\end{tabular}




\begin{tabular}{|c|c|c|}
\hline & & GitHub \\
\hline $\begin{array}{l}\text { Frictionless Data for } \\
\text { Reproducible Research Fellows } \\
\text { Programme }\end{array}$ & $\begin{array}{l}\text { https://fellows.frictionlessdata.i } \\
\text { o/ }\end{array}$ & $\begin{array}{l}\text { Learners; contains lessons, } \\
\text { resources, and syllabus for a } \\
\text { fellowship program }\end{array}$ \\
\hline Data Carpentry for Biologists & $\begin{array}{l}\text { https://datacarpentry.org/seme } \\
\text { ster-biology/ }\end{array}$ & $\begin{array}{l}\text { Learners, instructors; semester } \\
\text { long syllabus and class } \\
\text { materials, lessons }\end{array}$ \\
\hline ProjectTIER & https://www.projecttier.org/ & $\begin{array}{l}\text { Learners, instructors; exercises, } \\
\text { workshops and programs, and } \\
\text { templates }\end{array}$ \\
\hline $\begin{array}{l}\text { Psychology's Credibility } \\
\text { Revolution course }\end{array}$ & $\begin{array}{l}\text { https://docs.google.com/presen } \\
\text { tation/d/e/2PACX- } \\
\text { 1vREwmeADm5WZCBfjAKyAkzY } \\
\text { C_cQb7xtYv6C7e_wBJXOBUhJ3IF } \\
\text { YOWABplb0XotJake0Xrh- } \\
\text { CC6g1Vwy/pub?start=false\&am } \\
\text { p;loop=false\&amp;delayms=999 } \\
\text { 99999\#slide=id.g950fb47fc8_0_ } \\
16\end{array}$ & $\begin{array}{l}\text { Learners; course by Carleton } \\
\text { College faculty member Julia } \\
\text { Strand with lectures, exercises, } \\
\text { and more }\end{array}$ \\
\hline $\begin{array}{l}\text { STAT 545: Data wrangling, } \\
\text { exploration, and analysis with R }\end{array}$ & https://stat545.com/ & $\begin{array}{l}\text { Learners; course by University } \\
\text { of British Columbia faculty } \\
\text { member Jennifer Bryan }\end{array}$ \\
\hline
\end{tabular}

\section{CONCLUSION}

Reproducibility educational needs may vary by discipline, but there are transdisciplinary common threads that can be explored at all levels of learning. The conference's themes are a snapshot of emergent practices that can be used to embed reproducibility education throughout a lifelong learning process.

\section{Funding}

Research Reproducibility 2020: Educating for Reproducibility was supported by the Department of Health and Human Services Office of Research Integrity (ORIIR190046). The content is solely the responsibility of the authors and does not necessarily represent the official views of the U.S. Department of Health \& Human Services.

\section{Disclosure of Interests}


Melissa Rethlefsen and Hao Ye are founders of the ReproducibiliTea journal club at the University of Florida. Katherine MacWilkinson is an employee of the University of Florida Conference Department. Hao Ye is a certified Carpentries instructor and is a member of the Code of Conduct Committee for Repro4Everyone.

\section{Ethics Review}

The evaluation of Research Reproducibility 2020 was approved exempt by the University of Florida Institutional Review Board (IRB202000619).

All Research Reproducibility 2020 speakers agreed to be filmed and the recordings publicly shared.

\section{References}

1. Kligyte V, Marcy RT, Sevier ST, et al. A qualitative approach to Responsible Conduct of Research $(\mathrm{RCR})$ training development: identification of metacognitive strategies. Science and engineering ethics. 2008;14(1):3-31. doi: 10.1007/s11948-007-9035-4.

2. Mumford MD, Connelly S, Brown RP, et al. A Sensemaking Approach to Ethics Training for Scientists: Preliminary Evidence of Training Effectiveness. Ethics \& Behavior. 2008;18(4):315-39. doi: 10.1080/10508420802487815.

3. Baker M. 1,500 scientists lift the lid on reproducibility. Nature. 2016;533(7604):452-4. doi: DOI: http://dx.doi.org/10.1038/533452a.

4. Collins FS, Tabak LA. NIH plans to enhance reproducibility. Nature. 2014;505(7485):612-3. doi: DOI: http://dx.doi.org/10.1038/505612a.

5. Harris RF. Rigor mortis : how sloppy science creates worthless cures, crushes hope, and wastes billions. New York: Basic Books; 2017.

6. National Academies of Sciences Engineering and Medicine. Fostering Integrity in Research. Washington, DC: National Academies Press; 2017.

7. Button KS, loannidis JPA, Mokrysz C, et al. Power failure: why small sample size undermines the reliability of neuroscience. Nature Reviews Neuroscience. 2013;14:365. doi: 10.1038/nrn3475.

8. Ioannidis J, Doucouliagos C. What's to know about the credibility of empirical economics? Journal of Economic Surveys. 2013;27(5):997-1004. doi: 10.1111/joes.12032.

9. Dominus S. When the revolution came for Amy Cuddy. New York Times Magazine. 2017:28-55. 10. Lee SM. Sliced \& diced: Here's how Cornell scientist Brian Wansink turned shoddy data into viral studies about how we eat. Buzzfeed [Internet]. 2018. Available from: https://www.buzzfeednews.com/article/stephaniemlee/brian-wansink-cornell-p-hacking.

11. Marcus A, Oransky I. The data thugs. Science. 2018;359(6377):730-2. Epub 2018/02/17. doi: 10.1126/science.359.6377.730.

12. Bartlett T. 'I Want to Burn Things to the Ground'. Chronicle of Higher Education. 2018;65(3):1-. 13. Yong E. How the GOP could use science's reform movement against it. 2017 [cited 2017 May 24]. Available from: https://www.theatlantic.com/science/archive/2017/04/reproducibility-scienceopen-judoflip/521952/.

14. Fanelli D. How Many Scientists Fabricate and Falsify Research? A Systematic Review and MetaAnalysis of Survey Data. PLoS One. 2009;4(5):e5738. doi: 10.1371/journal.pone.0005738. 
15. Head ML, Holman L, Lanfear R, et al. The extent and consequences of $p$-hacking in science. PLoS biology. 2015;13(3):e1002106. Epub 2015/03/15. doi: 10.1371/journal.pbio.1002106.

16. Kerr NL. HARKing: hypothesizing after the results are known. Personality and Social Psychology Review. 1998;2(3):196-217. Epub 2005/01/14. doi: 10.1207/s15327957pspr0203_4.

17. Nosek B. Strategy for culture change. Charlottesville, VA: Center for Open Science; 2019 [updated 11 June; cited 202125 June]. Available from: https://www.cos.io/blog/strategy-for-culturechange.

18. Socio-Technical Centre. Socio-technical systems theory. Leeds, UK: University of Leeds; 2021 [cited 202125 June]. Available from: https://business.leeds.ac.uk/research-stc/doc/socio-technicalsystems-theory.

19. Erway R. Starting the conversation: University-wide research data management policy. 2013 [updated 6 Dec; cited 202125 June]. Available from: https://er.educause.edu/articles/2013/12/startingthe-conversation-universitywide-research-data-management-policy.

20. Pawlik A, van Gelder CWG, Nenadic A, et al. Developing a strategy for computational lab skills training through Software and Data Carpentry: Experiences from the ELIXIR Pilot action. F1000Research. 2017;6. Epub 2017/08/07. doi: 10.12688/f1000research.11718.1.

21. Teal TK, Cranston KA, Lapp H, et al. Data carpentry: workshops to increase data literacy for researchers. International Journal of Digital Curation. 2015;10(1):135-43.

22. Wilson G. Software Carpentry: lessons learned. F1000Research. 2014;3:62. Epub 2014/04/10. doi: 10.12688/f1000research.3-62.v2.

23. Research Reproducibility 2020. Gainesville, FL: University of Florida; 2020 [cited 202125 Jun]. Available from: https://pwd.aa.ufl.edu/researchre-pro/.

24. Nosek B. Culture change toward more open, rigorous, and reproducible research. Research Reproducibility 2020: Educating for Reproducibility; 2 Dec; Gainesville, FL2020. Available from: https://www.youtube.com/watch?v=FIYffIOmKI4; https://www.youtube.com/watch?v=0GmKhPGbNCM.

25. Ball R, Riegelman A, Teal T. Learning across disciplines: approaches to develop reproducibility education: Post-graduate and professional education. Research Reproducibility 2020: Educating for Reproducibility; 3 Dec; Gainesville, FL2020. Available from:

https://www.youtube.com/watch?v=Hv4qqYe5NOo.

26. Avery B, Gurka M, Millman KJ, et al. Learning across disciplines: approaches to develop reproducibility education: undergraduate / graduate education. Research Reproducibility 2020: Educating for Reproducibility; 2 Dec; Gainesville, FL2020. Available from: https://www.youtube.com/watch?v=YnuwSPGKfNs.

27. Allen MA, Cistola A, Cohen S, et al. Student Experience \& Support. Research Reproducibility 2020: Educating for Reproducibility; 3 Dec; Gainesville, FL2020. Available from: https://www.youtube.com/watch?v=3Mq8dx0HOK4.

28. Wickes E. Centering users within best practices: let "good enough" be enough. Research Reproducibility 2020: Educating for Reproducibility; 2 Dec; Gainesville, FL2020. Available from: https://pwd.aa.ufl.edu/researchre-pro/wp-content/uploads/sites/8/2020/11/Wickes.pdf.

29. Mears R. Replication of psychology experiments: engaging scientific and statistical reasoning in the classroom. Research Reproducibility 2020: Educating for Reproducibility; 2 Dec; Gainesville, FL2020. Available from: https://pwd.aa.ufl.edu/researchre-pro/wp-content/uploads/sites/8/2020/11/Poster 63 Mears Ryan.pdf.

30. Burr L, Goldsmith FH, Cowan M. Intelligibility, comprehensibility, and accentedness in L2 speech replication study. Research Reproducibility 2020: Educating for Reproducibility; 2 Dec; Gainesville, FL2020. Available from: https://pwd.aa.ufl.edu/researchre-pro/wpcontent/uploads/sites/8/2020/11/Poster 6-5 Burr Lindsi-2.pdf. 
31. Lapato D, Exner N. Broad engagement, collaboration, and teaching reproducibility skills in biomedical research. Research Reproducibility 2020: Educating for Reproducibility; 2 Dec; Gainesville, FL2020. Available from: https://www.youtube.com/watch?v=84yqr23Tic8.

32. Dunleavy DJ, Lacasse JR. Towards reproducible social work research: a graduate course on reproducibility, rigor, and meta-science. Research Reproducibility 2020: Educating for Reproducibility; 2 Dec; Gainesville, FL2020. Available from: https://www.youtube.com/watch?v=coRA67NNaiw.

33. Granell C, Sileryte R, Nüst D. Reproducible graduate theses in GIScience. Research Reproducibility 2020: Educating for Reproducibility; 2 Dec; Gainesville, FL2020. Available from: https://www.youtube.com/watch?v=abHlgYzLWP4.

34. LaPolla F, Surkis A. Developing a replicability workshop for our community of academic medical library users. Research Reproducibility 2020: Educating for Reproducibility; 2 Dec; Gainesville, FL2020. Available from: https://www.youtube.com/watch?v=62Irssu1KaU.

35. Chen $\mathrm{S}$, Chou W, Kung $\mathrm{C}$, et al. Building collaboration framework in countries replication crisis yet happened. Research Reproducibility 2020: Educating for Reproducibility; 2 Dec; Gainesville, FL2020. Available from: https://pwd.aa.ufl.edu/researchre-pro/wp-content/uploads/sites/8/2020/11/Poster 62 Chen Sau-Chin.pdf.

36. Johnson J, Goodman KW. From compliance to creativity: struggling to maintain the integrity of research integrity. Research Reproducibility 2020: Educating for Reproducibility; 2 Dec; Gainesville, FL2020. Available from: https://www.youtube.com/watch?v=-aeE8NVMZLA.

37. Winfree $L$, Barratt J. Lessons from the frictionless data for reproducible research fellows programme. Research Reproducibility 2020: Educating for Reproducibility; 2 Dec; Gainesville, FL2020. Available from: https://www.youtube.com/watch?v=b82Gp5akses.

38. Taylor BP, Marsiske M. Modeling good clinical practice principles from clinical trials to improve rigor, reproducibility, and transparency in single laboratory studies: an example from ACTIVE. Research Reproducibility 2020: Educating for Reproducibility; 2 Dec; Gainesville, FL2020. Available from: https://pwd.aa.ufl.edu/researchre-pro/wp-content/uploads/sites/8/2020/11/Poster 66 Taylor Brad.pdf.

39. Johnson G, Pedersen S, Pfahler A, et al. Using web-based experiments to support transparent and reproducible research. Research Reproducibility 2020: Educating for Reproducibility; 2 Dec; Gainesville, FL2020. Available from: https://www.youtube.com/watch?v=6MeynktRUXM.

40. Gottlieb G. Online training and badges to incentivize transparent and reproducible research. Research Reproducibility 2020: Educating for Reproducibility; 2 Dec; Gainesville, FL2020. Available from: https://www.youtube.com/watch?v=-Gyfw68-774.

41. Zaringhalam $M$, Federer L. Identifying barriers to reproducibility: an ethnographic study of a hands-on workshop. Research Reproducibility 2020: Educating for Reproducibility; 2 Dec; Gainesville, FL2020. Available from: https://www.youtube.com/watch?v=ojG2VITMmTI.

42. Abitua A, Bachle SM. Collaborative initiatives driven by a biorepository to support reproducible research. Research Reproducibility 2020: Educating for Reproducibility; 2 Dec; Gainesville, FL2020. Available from: https://pwd.aa.ufl.edu/researchre-pro/wp-content/uploads/sites/8/2020/11/Poster 61 Abitua Angela-1.pdf.

43. Reynolds P. 'Preproducibility' and backwards design: Improving preclinical research reproducibility with reporting guidelines. Research Reproducibility 2020: Educating for Reproducibility; 2 Dec; Gainesville, FL2020. Available from: https://www.youtube.com/watch?v=fFHC9glL8hc.

44. Selfe TK. Using reporting standards to promote research replicability and rigor in clinical trials. Research Reproducibility 2020: Educating for Reproducibility; 2 Dec; Gainesville, FL2020. Available from: https://pwd.aa.ufl.edu/researchre-pro/wp-content/uploads/sites/8/2020/11/Poster Selfe Terry.pdf.

45. Menke J, Roelandse M, Ozyurt B, et al. Rigor and Transparency Index, a new metric of quality for assessing biological and medical science methods. Research Reproducibility 2020: Educating for 
Reproducibility; 2 Dec; Gainesville, FL2020. Available from: https://pwd.aa.ufl.edu/researchre-pro/wpcontent/uploads/sites/8/2020/11/Poster 5-1 Bandrowski Anita.pdf.

46. Cooper K, Scherer L, Donahue M, et al. Transparent manuscript reporting practices in behavioral medicine research: An audit of publications in 2018. Research Reproducibility 2020: Educating for Reproducibility; 2 Dec; Gainesville, FL2020. Available from: https://pwd.aa.ufl.edu/researchre-pro/wpcontent/uploads/sites/8/2020/11/Poster 5-3 Cooper Kellie.pdf.

47. Eckmann P, Riedel N, Kilicoglu H, et al. Automated screening of COVID-19 preprints: can we help authors to improve transparency \& reproducibility? Research Reproducibility 2020: Educating for Reproducibility; 2 Dec; Gainesville, FL2020. Available from:

https://www.youtube.com/watch?v=mBAxIVE4KX8.

48. Meghreblian B, Chambers C, Tzavella L. Encouraging registered reports - metascience and tool development. Research Reproducibility 2020: Educating for Reproducibility; 2 Dec; Gainesville, FL2020. Available from: https://pwd.aa.ufl.edu/researchre-pro/wpcontent/uploads/sites/8/2020/11/Poster Meghreblian Ben.pdf.

49. 2020 Research Reproducibility Conference playlist. Gainesville, FL: University of Florida; 2020 [cited 202125 Jun]. Available from:

https://www.youtube.com/watch?v=RVBnbvV3VzQ\&list=PLIgMqnaPv2sw0va3GFk-abdfQ-PIwqVQL. 50. Research Reproducibility 2020 Educating for Reproducibility: Pathways to Research Integrity. Charlottesville, VA: OSFMeetings; 2020 [cited 202125 June]. Available from: https://osf.io/meetings/ResearchReproducibility2020/.

51. Research Reproducibility 2020: Agenda. Gainesville, FL: University of Florida; 2020 [cited 2021 25 Jun]. Available from: https://pwd.aa.ufl.edu/researchre-pro/agenda/.

52. Research_Reproducibility2020_Notes. 2020 [cited 202125 June]. Available from: https://docs.google.com/document/d/10yieZGXAjA7rKonVngpczGR-

2rvyRoJz5nvxwaCz754/edit?usp=sharing. 